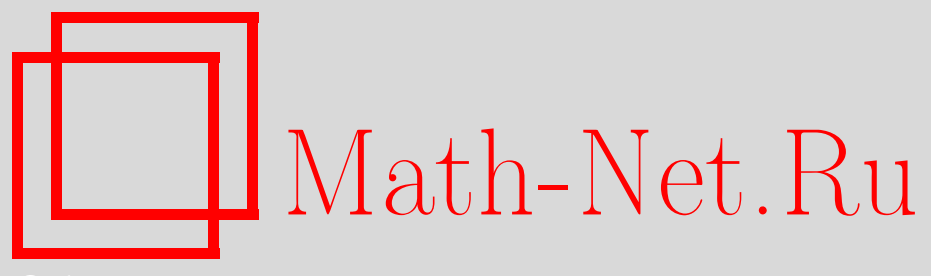

М. В. Долгополов, И. Н. Родионова, Задачи для уравнений гиперболического типа на плоскости и в трехмерном пространстве с условиями сопряжения на характеристике, Изв. РАН. Сер. матем., 2011, том 75, выпуск 4, 21-28

DOI: https://doi.org/10.4213/im4117

Использование Общероссийского математического портала Math-Net.Ru подразумевает, что вы прочитали и согласны с пользовательским соглашением http://www . mathnet.ru/rus/agreement

Параметры загрузки:

IP: 54.89 .56 .158

26 апреля 2023 г., 03:08:47

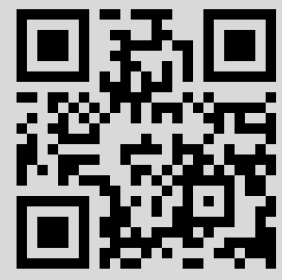




\author{
М. В. Долгополов, И. Н. Родионова
}

\title{
Задачи для уравнений гиперболического типа на плоскости и в трехмерном пространстве с условиями сопряжения на характеристике
}

\begin{abstract}
Для уравнения гиперболического типа на плоскости и одного из его аналогов третьего порядка в трехмерном пространстве во введенных авторами специальных классах решаются краевые задачи в областях, содержащих две линии (плоскости) сингулярности коэффициентов рассматриваемых уравнений, с условиями сопряжения на характеристической линии (плоскости).

Библиография: 3 наименования.

Ключевые слова: интегральные уравнения, краевые задачи, уравнения гиперболического типа.
\end{abstract}

\section{Введение}

Настоящая работа является продолжением исследований по теме "Специальные представления решений уравнений гиперболического типа и их приложений” [1]. В работах [1]-[3] для уравнения

$$
U_{\xi \eta}+\frac{p}{\eta-\xi} U_{\xi}-\frac{p}{\eta-\xi} U_{\eta}-\lambda U=0
$$

и одного из его пространственных аналогов

$$
U_{x y z}-\frac{p}{x-y-z} U_{x z}+\frac{p}{x-y-z} U_{y z}-\lambda U_{z}=0
$$

методом Римана были получены решения соответственно задачи Коши и видоизмененной задачи Коши (задачи С) для случая $0<p<1 / 2$. Посредством интегрального задания одного из условий задачи Коши (задачи С) получено специальное представление решений этих задач, что позволило распространить формулу решения поставленных задач на случай отрицательных и равных нулю значений параметра $p$, а также значительно упростить саму формулу. В случае отрицательных значений $p$ формула решения задачи Коши имеет более сложную структуру.

В настоящей работе решаются краевые задачи в областях, ограниченных двумя линиями (плоскостями) сингулярности коэффициентов рассматриваемых уравнений и условиями сопряжения на характеристической линии (плоскости) для случая отрицательных значений параметра $p$.

В $\S 1$ рассмотрено уравнение, полученное объединением уравнения (0.1) с уравнением, симметричным ему относительно оси $\eta=0$. Условия сопряжения для характеристики $\eta=0$ содержат производные, взятые по направлению нормалей к линиям сингулярности коэффициентов уравнения (задача $\mathrm{S}$ ). B $\S 2$ рассматривается один из пространственных аналогов задачи $\mathrm{S}$.

Работа выполнена при финансовой поддержке грантом АВЦП 3341 и 10854 Министерства образования и науки РФ.

(C) М. В. Долгополов, И. Н. РодионовА, 2011 


\section{§1. Постановка задачи}

Уравнение

$$
U_{\xi \eta}-\frac{p U_{\xi}}{\eta-\xi \operatorname{sign} \eta}+\frac{p U_{\eta}}{\eta \operatorname{sign} \eta-\xi}-\lambda U \operatorname{sign} \eta=0,
$$

где $0<p<1 / 2, p, \lambda$ - const, рассмотрим на множестве $D=D_{1} \cup D_{2}$; здесь $D_{1}=\{(\xi, \eta) \mid 0<\eta<\xi<h\}, h$ - const, $h>0, D_{2}=\{(\xi, \eta) \mid 0<-\eta<\xi<h\}$. В областях $D_{1}$ и $D_{2}$ уравнение (1.1) имеет соответственно вид

$$
\begin{aligned}
& U_{\xi \eta}-\frac{p}{\eta-\xi} U_{\xi}+\frac{p}{\eta-\xi} U_{\eta}-\lambda U=0, \\
& U_{\xi \eta}-\frac{p}{\eta+\xi} U_{\xi}-\frac{p}{\eta+\xi} U_{\eta}+\lambda U=0 .
\end{aligned}
$$

Аналогично [1] получим специальные представления решений задачи Коши для уравнения (1.1) в областях $D_{1}$ и $D_{2}$.

Решение задачи Коши для уравнения (1.1) в области $D_{1}$ с данными

$$
\begin{gathered}
\lim _{\eta \rightarrow \xi+0} U(\xi, \eta)=\tau_{1}(\xi), \quad 0 \leqslant \xi \leqslant h, \\
\lim _{\eta \rightarrow \xi+0}(\xi-\eta)^{-2 p}\left(U_{\xi}-U_{\eta}\right)=\nu_{1}(\xi), \quad 0<\xi<h,
\end{gathered}
$$

где $\tau_{1}^{\prime}(\xi) \in C[0, h], \nu_{1}(\xi) \in C(0, h)$ и $\nu_{1}(\xi)$ интегрируема на $[0, h]$, имеет вид

$$
\begin{aligned}
U(\xi, \eta)= & \int_{\xi}^{h} T_{1}(s)(s-\xi)^{p}(s-\eta)^{p}{ }_{0} F_{1}(1+p, \lambda(s-\eta)(s-\xi)) d s \\
& +\int_{\eta}^{\xi} N_{1}(s)(\xi-s)^{p}(s-\eta)^{p}{ }_{0} F_{1}(1+p,-\lambda(s-\eta)(\xi-s)) d s
\end{aligned}
$$

здесь

$$
\begin{gathered}
{ }_{0} F_{1}(\alpha, z)=\sum_{n=0}^{\infty} \frac{z^{n}}{(\alpha)_{n} n !}, \\
N_{1}(s)=\frac{1}{2 \cos (\pi p)} T_{1}(s)-\frac{1}{2} \frac{\Gamma(1+2 p)}{\Gamma^{2}(1+p)} \nu_{1}(s),
\end{gathered}
$$

если заданная функция $\tau_{1}(\xi)$ имеет интегральное представление

$$
\tau_{1}(\xi)=\int_{\xi}^{h} T_{1}(s)(s-\xi)^{2 p}{ }_{0} F_{1}\left(1+p, \lambda(s-\xi)^{2}\right) d s .
$$

Аналогично, в области $D_{2}$ решение задачи Коши с данными

$$
\begin{gathered}
\lim _{-\eta \rightarrow \xi+0} U(\xi, \eta)=\tau_{2}(\xi), \quad 0 \leqslant \xi \leqslant h, \\
\lim _{-\eta \rightarrow \xi+0}(\xi+\eta)^{-2 p}\left(U_{\eta}+U_{\xi}\right)=\nu_{2}(\xi), \quad 0<\xi<h,
\end{gathered}
$$

где $\tau_{2}^{\prime}(\xi) \in C[0, h], \nu_{2}(\xi) \in C(0, h)$ и $\nu_{2}(\xi)$ интегрируема на $[0, h]$, приводится к виду

$$
\begin{gathered}
U(\xi, \eta)=\int_{\xi}^{h} T_{2}(s)(s-\xi)^{p}(s+\eta)^{p}{ }_{0} F_{1}(1+p, \lambda(s+\eta)(s-\xi)) d s \\
+\int_{-\eta}^{\xi} N_{2}(s)(\xi-s)^{p}(s+\eta)^{p}{ }_{0} F_{1}(1+p,-\lambda(s+\eta)(\xi-s)) d s \\
N_{2}(s)=\frac{1}{\cos (\pi p)} T_{2}(s)+\frac{1}{2} \frac{\Gamma(1+2 p)}{\Gamma^{2}(1+p)} \nu_{2}(s)
\end{gathered}
$$


если функция $\tau_{2}(\xi)$ задается интегралом:

$$
\tau_{2}(\xi)=\int_{\xi}^{h} T_{2}(s)(s-\xi)^{2 p}{ }_{0} F_{1}\left(1+p, \lambda(s-\xi)^{2}\right) d s,
$$

где $T_{i}(s), i=1,2$, непрерывны в $(0, h)$ и интегрируемы на $[0, h]$.

Функции (1.3), (1.7) являются классическими решениями задачи Коши соответственно в областях $D_{1}$ и $D_{2}$, что доказывается непосредственной проверкой.

ЗАДАча S. На множестве $D$ найти решение уравнения (1.1), непрерывное в $\bar{D}$ и удовлетворяющее граничным условиям

$$
\begin{gathered}
U(h, \eta)=\varphi_{1}(\eta), \quad 0 \leqslant \eta \leqslant h, \\
U(h, \eta)=\varphi_{2}(\eta), \quad 0 \leqslant-\eta \leqslant h,
\end{gathered}
$$

с сопряжением на характеристике $\eta=0$ :

$$
\lim _{\eta \rightarrow 0+0}\left(\frac{\partial U}{\partial \eta}-\frac{\partial U}{\partial \xi}\right)=\lim _{\eta \rightarrow 0-0}\left(\frac{\partial U}{\partial \eta}+\frac{\partial U}{\partial \xi}\right) .
$$

На заданные функции $\varphi_{i}(\eta)$ налагаются следующие условия:

$$
\begin{gathered}
\varphi_{1}(\eta) \in C^{2}[0, h], \quad \varphi_{2}(\eta) \in C^{2}[-h, 0], \\
\varphi_{1}(h)=\varphi_{1}^{\prime}(h)=\varphi_{2}(-h)=\varphi_{2}^{\prime}(-h)=0, \\
\varphi_{i}(0)=\varphi_{i}^{\prime}(0)=0, \quad i=1,2 .
\end{gathered}
$$

Для решения поставленной задачи положим $\xi=h$ в формулах (1.3), (1.7) и подчиним функции условиям (1.9), (1.10). Получим следующие интегральные уравнения относительно $N_{i}(s), i=1,2$ :

$$
\begin{aligned}
& \int_{\eta}^{h} N_{1}(s)(h-s)^{p}(s-\eta)^{p}{ }_{0} F_{1}(1+p,-\lambda(s-\eta)(h-s)) d s=\varphi_{1}(\eta), \\
& \int_{-\eta}^{h} N_{2}(s)(h-s)^{p}(s+\eta)^{p}{ }_{0} F_{1}(1+p,-\lambda(s+\eta)(h-s)) d s=\varphi_{2}(\eta) .
\end{aligned}
$$

Рассуждениями, подобными тем, которые были проведены в работе [1], получаем единственное решение уравнений (1.14) и (1.15) соответственно:

$$
\begin{aligned}
N_{1}(x)= & \frac{(h-x)^{-p}}{\Gamma(1+p) \Gamma(1-p)}\left[\int_{x}^{h} \varphi_{1}^{\prime \prime}(s)(s-x)^{-p}{ }_{0} F_{1}(1-p, \lambda(s-x)(h-x)) d s\right. \\
& \left.\quad+\frac{\lambda}{1-p} \int_{x}^{h} \varphi_{1}^{\prime}(s)(s-x)^{1-p}{ }_{0} F_{1}(2-p, \lambda(s-x)(h-x)) d s\right], \quad(1.16) \\
N_{2}(x)= & \frac{(h-x)^{-p}}{\Gamma(1+p) \Gamma(1-p)}\left[\int_{x}^{h} \varphi_{2}^{\prime \prime}(-s)(s-x)^{-p}{ }_{0} F_{1}(1-p, \lambda(s-x)(h-x)) d s\right. \\
& \left.-\frac{\lambda}{1-p} \int_{x}^{h} \varphi_{2}^{\prime}(-s)(s-x)^{1-p}{ }_{0} F_{1}(2-p, \lambda(s-x)(h-x)) d s\right] .
\end{aligned}
$$

Делая в интегралах из формул (1.16), (1.17) замену $s=x+(h-x) \mu$ и учитывая непрерывность на $[0, h]$ функций $\varphi_{i}^{\prime \prime}(s)$ и ${ }_{0} F_{1}(\alpha, z)$, приходим к выводу, что при выполнении условий (1.12) функции $N_{i}(s)$ принадлежат $C[0, h]$. 
Для нахождения функций $T_{1}$ и $T_{2}$ воспользуемся непрерывностью решения задачи $\mathrm{S}$ на линии $\eta=0$ и условиями сопряжения (1.11), согласно которым получаем

$$
\begin{gathered}
\int_{\xi}^{\eta}\left[T_{1}(s)-T_{2}(s)\right] s^{p}(s-\xi)^{p}{ }_{0} F_{1}(1+p, \lambda s(s-\xi)) d s \\
=\int_{0}^{\xi}\left[N_{2}(s)-N_{1}(s)\right] s^{p}(\xi-s)^{p}{ }_{0} F_{1}(1+p, \lambda s(s-\xi)) d s \\
\int_{\xi}^{\eta}\left[T_{1}(s)+T_{2}(s)\right] s^{p}(s-\xi)^{p-1}{ }_{0} F_{1}(p, \lambda s(s-\xi)) d s \\
=\int_{0}^{\xi}\left[N_{2}(s)+N_{1}(s)\right] s^{p-1}(\xi-s)^{p-1}{ }_{0} F_{1}(p, \lambda s(s-\xi)) d s .
\end{gathered}
$$

Правые части уравнений $(1.18),(1.19)$ - известные функции, $N_{1}$ и $N_{2}$ в них определяются соответственно формулами (1.16), (1.17). Обозначим

$$
\begin{aligned}
\Phi_{1}(\xi) & \equiv \int_{0}^{\xi}\left[N_{2}(s)-N_{1}(s)\right] s^{p}(\xi-s)^{p}{ }_{0} F_{1}(1+p, \lambda s(s-\xi)) d s, \\
\Phi_{2}(\xi) & \equiv \int_{0}^{\xi}\left[N_{2}(s)+N_{1}(s)\right] s^{p-1}(\xi-s)^{p-1}{ }_{0} F_{1}(p, \lambda s(s-\xi)) d s .
\end{aligned}
$$

Для разрешимости уравнений (1.18), (1.19) в классе функций, непрерывных в $(0, h)$ и интегрируемых на $[0, h]$, необходимо, чтобы функции $\Phi_{i}(\xi)$ удовлетворяли условиям

$$
\Phi_{1}(h)=\Phi_{2}(h)=\Phi_{1}^{\prime}(h)=0, \quad \Phi_{1} \in C^{2}(0, h), \quad \Phi_{2} \in C^{1}(0, h),
$$

где $\Phi_{1}^{\prime \prime}$ и $\Phi_{2}^{\prime}$ интегрируемы на $[0, h]$.

Непосредственной проверкой показываем, что условия (1.22) будут выполняться, если к условиям (1.12), (1.13) добавить условие ортогональности

$$
\int_{0}^{h} \varphi_{i}(t) t d t=0, \quad i=1,2 .
$$

ЗАмЕчАНИЕ 1.1. Простейшим примером функции, удовлетворяющей условиям (1.12), (1.13), (1.23), служит

$$
\varphi(t)=t^{\alpha-1}(h-t)^{\beta}-t^{\beta-1}(h-t)^{\alpha}, \quad \alpha>2, \quad \beta>2 .
$$

Проводя вычисления, покажем, что если $\varphi_{i}(\eta) \in C_{[0, h]}^{(2)}$, то функции $\Phi_{1}^{\prime \prime}(\xi)$ и $\Phi_{2}^{\prime}(\xi)$ непрерывны на $(0, h]$, а в точке $\xi=0$ имеют особенность порядка $1-p$.

Основываясь на результатах работы [1], складывая и вычитая единственные решения уравнений (1.18), (1.19), приходим к выражениям

$$
\begin{gathered}
T_{1}(x)=\frac{x^{-p}}{2 \Gamma(p) \Gamma(1-p)}\left[\int_{x}^{h}\left(\frac{1}{p} \Phi_{1}^{\prime \prime}(s)-x \Phi_{2}^{\prime}(s)\right)(s-x)_{0}^{-p} F_{1}(1-p,-\lambda(s-x) x) d s\right. \\
\left.\quad+\frac{\lambda}{1-p} \int_{x}^{h}\left(\frac{1}{p} \Phi_{1}^{\prime}(s)-x \Phi_{2}(s)\right)(s-x)^{1-p}{ }_{0} F_{1}(2-p,-\lambda(s-x) x) d s\right],
\end{gathered}
$$




$$
\begin{aligned}
T_{2}(x)=- & \frac{x^{-p}}{2 \Gamma(p) \Gamma(1-p)}\left[\int_{x}^{h}\left(\frac{1}{p} \Phi_{1}^{\prime \prime}(s)+x \Phi_{2}^{\prime}(s)\right)(s-x)^{-p}{ }_{0} F_{1}(1-p,-\lambda(s-x) x) d s\right. \\
& \left.+\frac{\lambda}{1-p} \int_{x}^{h}\left(\frac{1}{p} \Phi_{1}^{\prime}(s)+x \Phi_{2}(s)\right)(s-x)^{1-p}{ }_{0} F_{1}(2-p,-\lambda(s-x) x) d s\right] .
\end{aligned}
$$

Проверкой устанавливаем, что функции (1.24), (1.25) при выполнении условий (1.22) непрерывны на $(0, h)$ и интегрируемы на $[0, h]$.

Из изложенного выше следует

Теорема 1.1. Задача $\mathrm{S}$ для уравнения (1.1) имеет единственное решение. При выполнении условий (1.12), (1.13), (1.23) решение задачи определяется формулами (1.3), (1.7), (1.16), (1.17), (1.24), (1.25).

ЗАмечАниЕ 1.2. Единственность решения задачи $\mathrm{S}$ следует из единственности решения задачи Коши, взятого за основу, а также из однозначной разрешимости всех интегральных уравнений, участвующих в процессе решения.

\section{§ 2. Пространственная задача MS}

Уравнение

$U_{x y z}+\frac{2 p y}{(x-z)^{2}-y^{2}} U_{x z}-\frac{2 p(x-z)}{(x-z)^{2}-y^{2}} U_{y z}-4 \lambda y(x-z) U_{z} \operatorname{sign} y=0, \quad 0<p<\frac{1}{2}$,

рассмотрим на множестве $\mathcal{G}=\mathcal{G}_{1} \cup \mathcal{G}_{2}$, где

$$
\begin{gathered}
\mathcal{G}_{1}=\{(x, y, z) \mid 0<z<x-y, 0<y<x<h\}, \\
\mathcal{G}_{2}=\{(x, y, z) \mid 0<z<x+y, 0<-y<x<h\}, \quad h>0 .
\end{gathered}
$$

Части $z=x-y$ и $z=x+y$ границы $\mathcal{G}$ являются плоскостями сингулярности коэффициентов уравнения (2.1).

Решение видоизмененной задачи Коши (задачи С) для уравнения (2.1) с данными

$$
\begin{gathered}
U(x, y, x-y)=\tau_{1}(x, y), \quad 0 \leqslant x \leqslant y \leqslant h, \\
\lim _{z \rightarrow x-y-0}(x-y-z)^{-2 p}(2 y)^{-2 p}\left(U_{x z}-U_{y z}\right)=\mu_{1}(x, y), \quad 0<x<y<h, \\
\lim _{z \rightarrow x-y-0} \frac{\partial U}{\partial z}=\nu_{1}(x, y), \quad 0<x<y<h,
\end{gathered}
$$

где $\mu_{1}(x, y), \nu_{1}(x, y)$ непрерывны в области $\Delta_{1}=\{x, y \mid 0<x<y<h\}$ и интегрируемы в $\bar{\Delta}_{1},\left(\tau_{1}\right)_{x y} \in C\left(\bar{\Delta}_{1}\right)$, получено в работе [3] и в силу интегрального представления функции $\nu_{1}$ :

$\nu_{1}(x, x-y)=\int_{x}^{h} T_{1}(\xi, x-y)\left[(\xi-x+y)^{2}-y^{2}\right]^{2 p}{ }_{0} F_{1}\left(1+p,-\lambda\left[(\xi-x+y)^{2}-y^{2}\right]^{2}\right) d \xi$,

где $T_{1}$ - непрерывная в $\Delta_{1}$ и интегрируемая в $\bar{\Delta}_{1}$ функция, приводится к виду

$$
\begin{array}{rl}
U(x, y, z)=\tau_{1}(x, y)-\int_{z}^{x-y} & d s \int_{y+s}^{x} N_{1}(\xi, s)\left[(\xi-s)^{2}-y^{2}\right]^{p}\left[(x-s)^{2}-(\xi-s)^{2}\right]^{p} \\
& \times{ }_{0} F_{1}\left(1+p, \lambda\left[(\xi-s)^{2}-y^{2}\right]\left[(x-s)^{2}-(\xi-s)^{2}\right]\right) d \xi
\end{array}
$$




$$
\begin{array}{rl}
-\int_{z}^{x-y} & d s \int_{x}^{h} T_{1}(\xi, s)\left[(\xi-s)^{2}-y^{2}\right]^{p}\left[(\xi-s)^{2}-(x-s)^{2}\right]^{p} \\
& \times{ }_{0} F_{1}\left(1+p, \lambda\left[(\xi-s)^{2}-y^{2}\right]\left[(x-s)^{2}-(\xi-s)^{2}\right]\right) d \xi .
\end{array}
$$

Аналогично получаем решение задачи С специального представления в области $\mathcal{G}_{2}$. Это функция

$$
\begin{aligned}
U(x, y, z)=\tau_{2}(x, y) & -\int_{z}^{x+y} d s \int_{s-y}^{x} N_{2}(\xi, s)\left[(\xi-s)^{2}-y^{2}\right]^{p}\left[(x-s)^{2}-(\xi-s)^{2}\right]^{p} \\
& \times{ }_{0} F_{1}\left(1+p, \lambda\left[(\xi-s)^{2}-y^{2}\right]\left[(x-s)^{2}-(\xi-s)^{2}\right]\right) d \xi \\
- & \quad \int_{z}^{x+y} d s \int_{x}^{h} T_{2}(\xi, s)\left[(\xi-s)^{2}-y^{2}\right]^{p}\left[(\xi-s)^{2}-(x-s)^{2}\right]^{p} \\
& \times{ }_{0} F_{1}\left(1+p, \lambda\left[(\xi-s)^{2}-y^{2}\right]\left[(x-s)^{2}-(\xi-s)^{2}\right]\right) d \xi
\end{aligned}
$$

удовлетворяющая в области $\mathcal{G}_{2}$ уравнению $(2.1)$ и граничным условиям

$$
\begin{array}{rlrl}
U(x, y, x+y) & =\tau_{2}(x, y), & 0 \leqslant x \leqslant-y \leqslant h, \\
\lim _{z \rightarrow x+y-0} \frac{\partial U}{\partial z}=\nu_{2}(x, y), & 0<x<-y<h,
\end{array}
$$

$\lim _{z \rightarrow x+y-0}(x+y-z)^{-2 p}(-2 y)^{-2 p}\left(U_{x z}+U_{y z}\right)=\mu_{2}(x, y), \quad 0<x<-y<h$,

где $\tau_{2}, \mu_{2}, \nu_{2}$ - заданные функции, $\mu_{2}, \nu_{2}$ непрерывны в $\Delta_{2}=\{(x, y) \mid 0<x<$ $-y<h\}$ и интегрируемы в $\bar{\Delta}_{2},\left(\tau_{2}\right)_{x y} \in C\left(\bar{\Delta}_{2}\right)$.

При этом функция $\nu_{2}$ имеет следующее представление:

$\nu_{2}(x, x+y)=\int_{x}^{h} T_{2}(\xi, x+y)\left[(\xi-x-y)^{2}-y^{2}\right]^{2 p}{ }_{0} F_{1}\left(1+p,-\lambda\left[(\xi-x-y)^{2}-y^{2}\right]^{2}\right) d \xi$,

в котором $T_{2}(x, y)$ - функция, непрерывная в $\Delta_{2}$ и интегрируемая в $\bar{\Delta}_{2}$.

В выражении (2.4) функция $N_{2}(\xi, s)$ определяется формулой

$$
N_{2}(\xi, s)=\frac{1}{2 \cos (\pi p)} T_{2}(\xi, s)+\frac{\Gamma(1+2 p)}{2 \Gamma^{2}(1+p)} \mu_{2}(\xi+s, s) .
$$

Непосредственными вычислениями доказывается, что функции (2.3), (2.4) при выполнении условий, налагаемых на функции $T_{i}, N_{i}, \tau_{i}, i=1,2$, удовлетворяют уравнению $(2.1)$ в областях $\mathcal{G}_{1}$ и $\mathcal{G}_{2}$ соответственно и условиям задачи С, а также являются непрерывными в замкнутых областях.

ЗАДАчА MS. На множестве $\mathcal{G}$ найти решение уравнения (2.1), непрерывное в $\overline{\mathcal{G}}$ и удовлетворяющее граничным условиям $(2.2),(2.5)$ и условию

$$
U(h, y, z)= \begin{cases}\varphi_{1}(y, z), & (y, z) \in \bar{\Delta}_{3}=\{(y, z) \mid 0 \leqslant y \leqslant h-z, 0 \leqslant z \leqslant h\} \\ \varphi_{2}(y, z), & (y, z) \in \bar{\Delta}_{3}^{*}=\{(y, z) \mid 0 \leqslant-y \leqslant h-z, 0 \leqslant z \leqslant h\}\end{cases}
$$

с сопряжением на плоскости $y=0$ :

$$
\lim _{y \rightarrow 0-0}\left(U_{y}-U_{x}\right)=\lim _{y \rightarrow 0+0}\left(U_{y}+U_{x}\right) .
$$


На заданные функции налагаются следующие условия:

$$
\begin{gathered}
\left(\tau_{1}\right)_{x y} \in C\left(\bar{\Delta}_{1}\right), \quad\left(\tau_{2}\right)_{x y} \in C\left(\bar{\Delta}_{2}\right), \\
\tau_{1}(h, y)=\tau_{2}(h, y)=0, \quad \tau_{1}(x, 0)=\tau_{2}(x, 0), \\
\frac{\partial}{\partial x} \tau_{1}(x, 0)+\frac{\partial}{\partial y} \tau_{1}(x, 0)=\frac{\partial}{\partial y} \tau_{2}(x, 0)-\frac{\partial}{\partial x} \tau_{2}(x, 0), \\
\varphi_{1}(h-z, z)=\frac{\partial}{\partial z} \varphi_{1}(h-z, z)=\frac{\partial^{2} \varphi_{1}(h-z, z)}{\partial z \partial y}=0, \\
\varphi_{2}(z-h, z)=\frac{\partial}{\partial z} \varphi_{2}(z-h, z)=\frac{\partial^{2} \varphi_{2}(z-h, z)}{\partial z \partial y}=0 .
\end{gathered}
$$

Обозначим

$$
\begin{gathered}
\frac{\partial}{\partial y}\left[\frac{\partial^{2} \varphi_{1}(y, z)}{\partial z \partial y} \frac{1}{y}\right]=\Phi_{1}(y, z), \\
\frac{\partial}{\partial y}\left[\frac{\partial^{2} \varphi_{2}(-y, z)}{\partial z \partial y} \frac{1}{y}\right]=\Phi_{2}(y, z), \\
\Phi_{1}(y, z) \in C\left(\bar{\Delta}_{3}\right), \quad \Phi_{2}(y, z) \in C\left(\bar{\Delta}_{3}^{*}\right) .
\end{gathered}
$$

Решение задачи MS технически не отличается от решения ее плоского аналога, поэтому сформулируем основные результаты. Условия (2.6) приводят к интегральным уравнениям относительно $N_{1}, N_{2}$, единственное решение которых при выполнении условий (2.9)-(2.15) имеет представление

$$
\begin{aligned}
& N_{1}(\xi, s)=\frac{\left[(h-s)^{2}-(\xi-s)^{2}\right]^{-p}}{\Gamma(1-p) \Gamma(1+p)}\left[(\xi-s) \int_{\xi-s}^{h-s}\left(\frac{\left(\varphi_{1}\right)_{z t}^{\prime \prime}(t, z)}{t}\right)_{t}^{\prime}\left[t^{2}-(\xi-s)^{2}\right]^{-p}\right. \\
& \times{ }_{0} F_{1}\left(1-p,-\lambda\left[t^{2}-(\xi-s)^{2}\right]\left[(h-s)^{2}-(\xi-s)^{2}\right]\right) d t \\
& +\frac{2 \lambda}{1-p} \int_{\xi-s}^{h-s}\left(\varphi_{1}\right)_{s t}^{\prime \prime}(t, s)\left[t^{2}-(\xi-s)^{2}\right]^{1-p} \\
& \left.\times{ }_{0} F_{1}\left(2-p,-\lambda\left[t^{2}-(\xi-s)^{2}\right]\left[(h-s)^{2}-(\xi-s)^{2}\right]\right) d t\right], \\
& N_{2}(\xi, s)=-\frac{\left[(h-s)^{2}-(\xi-s)^{2}\right]^{-p}}{\Gamma(1-p) \Gamma(1+p)}\left[(\xi-s) \int_{\xi-s}^{h-s}\left(\frac{\left(\varphi_{2}\right)_{s t}^{\prime \prime}(-t, s)}{t}\right)_{t}^{\prime}\left[t^{2}-(\xi-s)^{2}\right]^{-p}\right. \\
& \times{ }_{0} F_{1}\left(1-p,-\lambda\left[t^{2}-(\xi-s)^{2}\right]\left[(h-s)^{2}-(\xi-s)^{2}\right]\right) d t \\
& +\frac{2 \lambda}{1-p} \int_{\xi-s}^{h-s}\left(\varphi_{2}\right)_{s t}^{\prime \prime}(-t, s)\left[t^{2}-(\xi-s)^{2}\right]^{1-p} \\
& \left.\times{ }_{0} F_{1}\left(2-p,-\lambda\left[t^{2}-(\xi-s)^{2}\right]\left[(h-s)^{2}-(\xi-s)^{2}\right]\right) d t\right] .
\end{aligned}
$$

Функции (2.16), (2.17) при выполнении условий (2.11)-(2.15) непрерывны в $\bar{\Delta}_{1}$. Непрерывность решения задачи MS на $y=0$ и условия сопряжения (2.7) приводит нас к интегральным уравнениям относительно $T_{1} \pm T_{2}$, решая которые, получаем

$$
\begin{aligned}
T_{1}(\xi, z)=\frac{(\xi-z)^{1-2 p}}{\Gamma(p) \Gamma(1-p)} & {\left[\frac{\lambda}{1-p} \int_{\xi-z}^{h-z}\left[\Psi_{1}(t, z)+\Psi_{2}(t, z)\right]\left(t^{2}-(\xi-z)^{2}\right)^{1-p}\right.} \\
\times & { }_{0} F_{1}\left(2-p, \lambda[\xi-z]^{2}\left[t^{2}-(\xi-z)^{2}\right]\right) d\left(t^{2}\right) \\
- & \int_{\xi-z}^{h-z}\left[\Psi_{1}(t, z)+\Psi_{2}(t, z)\right]_{t}^{\prime}\left(t^{2}-(\xi-z)^{2}\right)^{-p}
\end{aligned}
$$




$$
\begin{aligned}
\times & \left.{ }_{0} F_{1}\left(1-p, \lambda[\xi-z]^{2}\left[t^{2}-(\xi-z)^{2}\right]\right) d t\right] \\
T_{2}(\xi, z)=\frac{(\xi-z)^{1-2 p}}{\Gamma(p) \Gamma(1-p)} & {\left[\frac{\lambda}{1-p} \int_{\xi-z}^{h-z}\left[\Psi_{1}(t, z)-\Psi_{2}(t, z)\right]\left(t^{2}-(\xi-z)^{2}\right)^{1-p}\right.} \\
\times & { }_{0} F_{1}\left(2-p, \lambda[\xi-z]^{2}\left[t^{2}-(\xi-z)^{2}\right]\right) d\left(t^{2}\right) \\
& -\int_{\xi-z}^{h-z}\left[\Psi_{1}(t, z)-\Psi_{2}(t, z)\right]_{t}^{\prime}\left(t^{2}-(\xi-z)^{2}\right)^{-p} \\
& \left.\times{ }_{0} F_{1}\left(1-p, \lambda[\xi-z]^{2}\left[t^{2}-(\xi-z)^{2}\right]\right) d t\right]
\end{aligned}
$$

Функции $\Psi_{i}, i=1,2$, определены формулами

$$
\begin{aligned}
& \Psi_{1}(t, z) \equiv \int_{z}^{t+z}\left[N_{1}(\xi, z)+N_{2}(\xi, z)\right](\xi-z)^{2 p}\left[t^{2}-(\xi-z)^{2}\right]^{p-1} \\
& \times{ }_{0} F_{1}\left(p, \lambda[\xi-z]^{2}\left[t^{2}-(\xi-z)^{2}\right]\right) d \xi \\
& \Psi_{2}(t, z) \equiv-\int_{z}^{t+z}\left[N_{1}(\xi, z)-N_{2}(\xi, z)\right](\xi-z)^{2 p}\left[t^{2}-(\xi-z)^{2}\right]^{p-1} \\
& \times{ }_{0} F_{1}\left(p, \lambda[\xi-z]^{2}\left[t^{2}-(\xi-z)^{2}\right]\right) d \xi .
\end{aligned}
$$

Проведя вычисления, покажем, что при выполнении условий (2.8)-(2.15) функции $T_{i}(x, y)$ непрерывны в соответствующих областях $\Delta_{i}$ и интегрируемы в $\bar{\Delta}_{i}$, $i=1,2$.

ТЕОРема 2.1. При выполнении условий (2.8)-(2.15) задача MS для уравнения (2.1) имеет единственное решение, определяемое формулами (2.3), (2.4), $(2.16)-(2.21)$.

Отметим, что единственность решения задачи следует из единственности решения видоизмененной задачи Коши, полученного методом Римана и взятого за основу, а также из однозначной разрешимости всех интегральных уравнений, участвующих в процессе решения.

\section{Список литературы}

1. В. М. Долгополов, М. В. Долгополов, И.Н. Родионова, "Построение специальных классов решений некоторых дифференциальных уравнений гиперболического типа", Докл. РАН, 429:5 (2009), 583-589; англ. пер.: V. M. Dolgopolov, M. V. Dolgopolov, I. N. Rodionova, "Construction of special classes of solutions for some differential equations of hyperbolic type", Dokl. Math., 80:3 (2009), 860-866.

2. В. М. Долгополов, И. Н. Родионова Видоизмененная задача Коши для одного гиперболического уравнения третьего порядка в трехмерном пространстве, Вестн. Сам. гос. техн. ун-та. Сер. физ.-матем. наук, 1:18 (2009), 41-46.

3. В. М. Долгополов, М. В. Долгополов, И. Н. Родионова, “Две задачи для гиперболического уравнения в трехмерном пространстве", Вестн. Сам. гос. техн. ун-та. Сер. естеств., 67:8 (2008), 95-107.

M. В. Долгополов (M. V. Dolgopolov)

Поступило в редакцию

Самарский государственный университет 25.05 .2009

E-mail: mvdolg@ssu.samara.ru, 19.04.2010

mikhaildolgopolov@rambler.ru

И. Н. Родионова (I. N. Rodionova)

Самарский государственный университет 